\title{
Roles of Exosomes from Mesenchymal Stem Cells in Treating Osteoarthritis
}

\author{
Jian Wang,, Xuanxuan Guo, Zhanrong Kang, ${ }^{2}$ Lingbin Qi,, ${ }^{3}$ Ying Yang, ${ }^{4}$ Juan Wang, ${ }^{5}$ \\ Jun $\mathrm{Xu}^{1}$, and Shane Gao ${ }^{1}$
}

\begin{abstract}
Exosomes are small extracellular vesicles (EVs) with a diameter of 50-150 nm that play important roles in cellto-cell communication through transportation of proteins, microRNAs, lncRNAs, and mRNAs. Some components, such as miRNAs, have been proven to be involved in inflammation regulation. Osteoarthritis (OA) is a progressive disease resulting in articular cartilage degeneration and subchondral bone deficiency. Complicated relationships between the breakdown of extracellular matrix and inflammation make it difficult to recover thoroughly. Current studies reported that exosomes secreted by mesenchymal stem cells (MSCs) can change disease evolution and protect the cartilage matrix in OA. In addition, exosomes obtained from human adipose derived stem cells downregulate inflammation and oxidative stress, which might mediate antisenescence in OA. The goal of this review is to describe and summarize the role of mesenchymal stem cell (MSC)-derived exosomes in $\mathrm{OA}$, focusing on their potential mechanism and possible therapeutic strategies.
\end{abstract}

Keywords: osteoarthritis (OA), exosomes, human adipose derived stem cells (hADSCs), extracellular matrix (ECM)

\section{Introduction}

$\mathbf{T}$ HE KNEE JOINT has a complicated microenvironment consisting of many types of cells, such as osteocytes, hematopoietic stem cells, chondrocytes, synovial cells, nerve cells, and fat cells. Osteoarthritis (OA) is a progressive and heterogeneous disease that contributes to articular cartilage degeneration, alterations in subchondral bone, and synovial hyperplasia (Allen and Golightly, 2015; Martel-Pelletier et al., 2016). Therefore, reversing aberrant metabolism in the articular cavity may provide a novel strategy for OA treatment (Tofino-Vian et al., 2017). Diverse efforts have been devoted to treating this disease; however, a complete cure for OA has not been reported domestically or internationally. Ordinary clinical therapies can only relieve pain and delay functional loss through nondrug or drug treatment.

Recently much evidence has shown that mesenchymal stem cells (MSCs) act as a key element in the balance between homeostasis and destabilization in OA (Chiang et al., 2016; Gnecchi et al., 2016; Pak et al., 2016; Roelofs et al.,
2013). MSCs are multipotent stromal cells that can differentiate into various cell types, including chondrocytes, osteoblasts, adipocytes, and myocytes. A Korea laboratory carried out a pilot clinical trial by recruiting 18 patients with OA. They first isolated adipose derived stem cells (ADSCs) from the fat of the patients. Then, the prepared ADSCs were injected into the knees continuously. After 6 months treatment, patients experienced pain relief, and their ability to live independently was increased compared with that of the placebo group (Jo et al., 2014).

Another clinical study indicated that the best therapeutic effect was achieved by injecting $5 \times 10^{7}$ ADSCs into the pathological tissue (Liu et al., 2017a). A rabbit experiment showed that allogenic bone marrow-mesenchymal stem cell (BM-MSC) transplantation could prevent the progression of OA (Chiang et al., 2016). It has been inferred that MSCs can migrate to injury (Leibacher and Henschler, 2016). However, many preclinical studies have shown that administered cells fail to incorporate into damaged tissue and differentiate into specific cell types. Increasing data demonstrate that

\footnotetext{
${ }^{1}$ East Hospital, Tongji University School of Medicine, Shanghai, China.

${ }^{2}$ Department of Orthopaedics, Shanghai Pudong Hospital, Fudan University, Shanghai, China.

${ }^{3}$ Department of Regenerative Medicine, Tongji University School of Medicine, Shanghai, China.

${ }^{4}$ Key Lab of Glycoconjugate Research, Department of Biochemistry and Molecular Biology, School of Basic Medical Sciences, Fudan University, Ministry of Public Health, Shanghai, China.

${ }^{5}$ Department of Cell Engineering, Binzhou Medical College, Yantai, China.
} 
MSCs exert their therapeutic effects through paracrine effects (Gnecchi et al., 2016) especially exosomes (Kordelas et al., 2014; Witwer et al., 2019).

The definition and source of exosomes are described in detail in the MISEV2014 guidelines and the updated MISEV2018 guidelines (Lotvall et al., 2014; Thery et al., 2018). Exosomes were identified more than 30 years ago in reticulocytes (Johnstone et al., 1987) and initially regarded as carriers of unwanted cellular components (Ludwig and Giebel, 2012). The current recognized definition of exosomes is nanoscale extracellular lipid bilayer vesicles derived from endocytic origin, which fuse with the plasma membrane of target cells, are internalized by endocytosis, or interact with cell surface receptors to induce intracellular signaling pathways (Iacopetta et al., 1983).

Exosomes can be secreted by various cell types, including reticulocytes (Johnstone et al., 1987), B cells (Blanchard et al., 2002), human adipose stem cell (Tofino-Vian et al., 2017), mast cells (Qin et al., 2016), and tumor cells (Gao et al., 2018). Although exosomes play key roles in maintaining homeostasis in organisms, they may also promote pathogenic progression by exerting detrimental effects. Exosomes originating from cancer stem cells contribute to tumor progression, therapy resistance, and tumor relapse by modulating the tumor microenvironment (Sharma, 2018). Whether exosomes generate physiological or pathogenic functions depends on their parental cells.

Recently, many efforts have been dedicated to determining how to isolate and identify exosomes (Table 1). The most commonly used method is differential centrifugation (DC) to eliminate precipitates and analogs of exosomes. However, the procedure is time consuming, and the purity is poor (Ailawadi et al., 2015). Recently, some commercial precipitation reagents, such as the ExoQuick and Total Exosome Isolation systems, have become available and have shown good specificity and yields (Ding et al., 2018). ExtraPEG and density gradient centrifugation are also common methods to isolate exosome (Momen-Heravi et al., 2013).

With the help of modern technology, novel technical isolation techniques, such as microfluidic viscoelastic flows (Liu et al., 2017a), asymmetric flow field-flow fractionation (Zhang et al., 2018), integrated double-filtration devices (Liang et al., 2017), size-exclusion chromatography (Böing et al., 2014), affinity-based methods (Nakai et al., 2016), and stochastic optical reconstruction microscopy
(Chen et al., 2016a), have been used to extract and characterize exosomes.

The following experiments need to be conducted to better identify exosomes (Fig. 1): nanoparticle tracking analysis, Western blot, and transmission electron microscopy. Nanoparticle tracking analysis is a method for characterization of particle diameter and volume depending on the light path variations induced by the particle. Because of the lack of visual particle morphology, this method cannot distinguish exosomes from protein polymers or nanoparticles. Western blot can identify some of the distinctive proteins accumulated in exosomes, but cell debris lysosomes and endosomes also contain tetraspanin, CD63, CD9, and ALIX. Although transmission electron microscopy can be used to analyze the structure of exosomes, mycoplasma, ferritin, polyethylene glycol protein polymers, and lysosomes have similar structures. Therefore, the above three experiments should be carried out in a complementary way.

\section{Exosomes in Osteoarthritis Diseases}

\section{Roles of MSC-exos in OA extracellular matrix}

The cartilage extracellular matrix (ECM) is a delicately organized network of various proteins and polysaccharides that are secreted into the extracellular space by chondrocytes and other cells. Chondrocytes synthesize and secrete a variety of matrix components, including collagen and glycosaminoglycans, as well as some matrix-degrading enzymes (Lories, 2008). While chondrocytes play a crucial role during the formation of ECM, their function is also tightly controlled by the ECM (Iliopoulos et al., 2008). The ECM is critical for sustaining the balance of articular cartilage, which has a low density of chondrocytes in a hypoxic environment (Withrow et al., 2016).

Proteins of the matrix metalloproteinase (MMP) family are involved in the breakdown of ECM during arthrosis pathologies, such as arthritis and tumor metastasis. Similarly, using a rabbit model, Kuroda et al. (2015) indicated that ADSCs could downregulate tumor necrosis factor- $\alpha$ (TNF- $\alpha$ )-mediated expression of MMP-13 by chondrocytes through paracrine effects. Consistently, after ADSC injection into the rabbit OA model, the expression of MMP-13 was found to be significantly decreased. MSC-exos could promote GAG synthesis affected by IL- $1 \beta$ and suppress IL$1 \beta$-induced nitric oxide (NO) and MMP-13 production. The

Table 1. Different Methods to Isolation of Exosomes

\begin{tabular}{|c|c|c|c|c|}
\hline Exosome isolation & Sample & Medium & Advantages & Reference \\
\hline ExtraPEG isolation & $\mathrm{BF}$ & PEG & Gentle & 19 \\
\hline Microfluidic viscoelastic flows & CCS, serum & PEO & High purity & 5 \\
\hline Ultracentrifugation & $\mathrm{BF}$ & $\mathrm{BF}$ & High recovery & 17 \\
\hline $\begin{array}{l}\text { Asymmetric flow field-flow } \\
\text { fractionation (AF4) }\end{array}$ & $\mathrm{BF}$ & Asymmetric flow & Highly reproducible & 21 \\
\hline Integrated double-filtration device & Urine & Microchip ELISA & Highly potential to clinical & 22 \\
\hline ExoQuick reagent & $\mathrm{BF}$ & EQ-precipitation solution & Convenient & 18 \\
\hline Total exosome isolation reagent & $\mathrm{BF}$ & Precipitation solution & High purity & 18 \\
\hline Size-exclusion chromatography & Plasma & Sepharose CL-2B & Quick and cheap & 23 \\
\hline affinity-based method & CCS & $\operatorname{Tim} 4$ & High purity & 24 \\
\hline density gradient centrifugation & $\mathrm{BF}$ & Sucrose & High purity & 20 \\
\hline
\end{tabular}

BF, biological fluid; CCS, cell culture supernatant; PEG, polyethylene glycol; PEO, poly-(oxyethylene); Tim4, T cell immunoglobulin domain and mucin domain-containing protein4. 


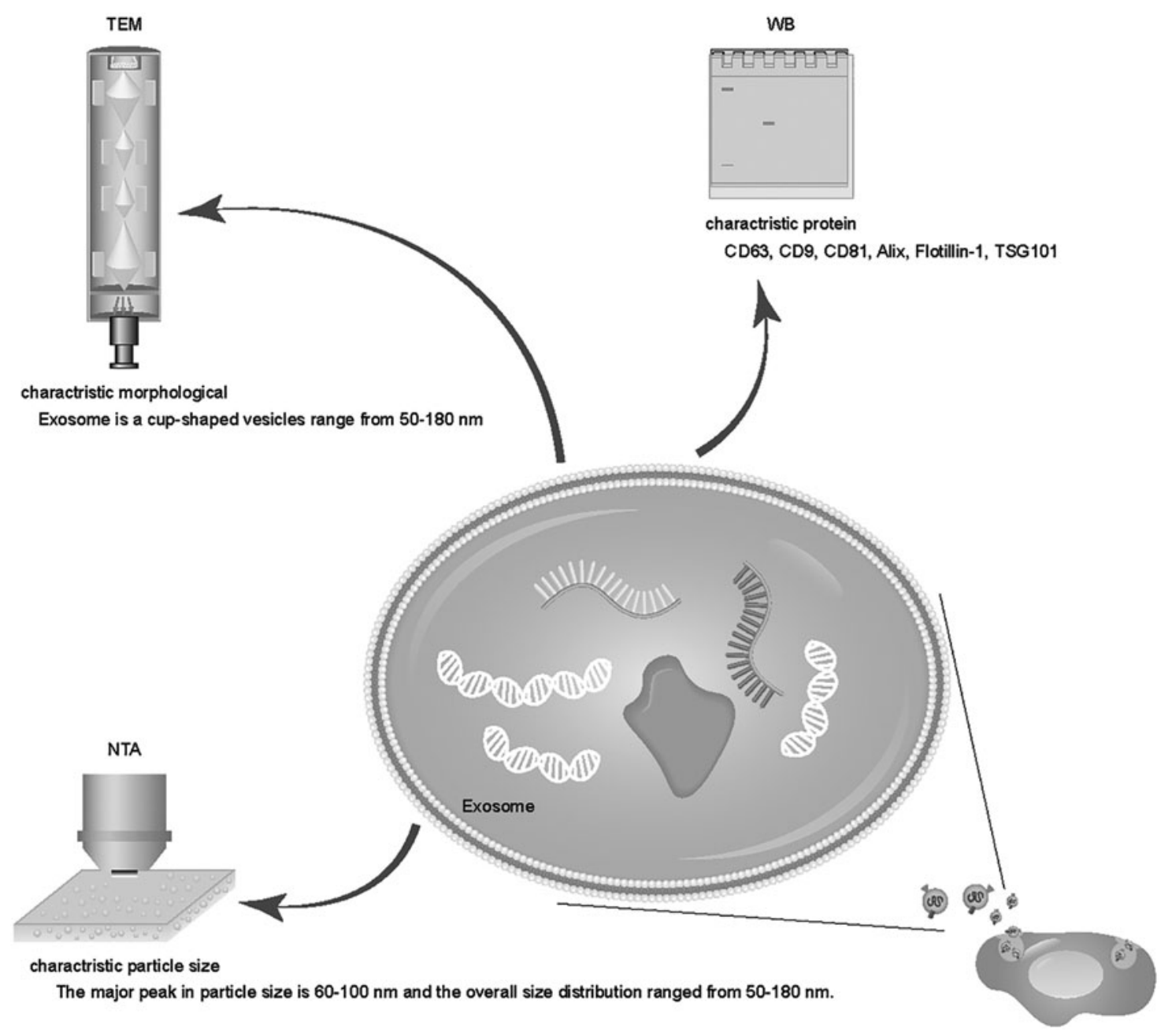

FIG. 1. Identify of exosomes. TEM, WB, and NTA are used to identify exosomes. NTA, nanosight tracking analysis; TEM, transmission electron microscopy; WB, western blot.

authors speculated that the modulation of the chondrocyte matrix was due to the contents of MSC-exos, especially proteins (Zhang et al., 2019).

Exosomes derived from synovial MSCs significantly boosted chondrocyte proliferation and migration but suppressed ECM protein synthesis. This suppression could be complemented by exosomes derived from miR-140-5poverexpressing synovial MSCs (SMSCs) (Tao et al., 2017).

\section{MSC-exos promote articular cartilage regeneration during $O A$}

Inducing self-healing of articular cartilage, which has limited blood vessels and nerves, is an enormous challenge. Some traditional clinical treatments do not manage to solve fibrosis, so they attempt to maintain a balance between fibrocartilage-like scar tissue and cambium (Makris et al., 2015). MSCs combined with scaffolds have been a prospective source for cartilage regeneration due to the chondrogenic ability of stem cells (Pittenger et al., 1999). Saw et al. (2013) showed that intra-articular injection of autologous peripheral blood stem cells combined with hyaluronic acid (HC) resulted in significantly higher cartilage production compared with parallel treatment with only HC.
In a rabbit OA model, implantation of poly-lactic coglycolic acid (PLGA) equipped with MSCs into the injured articular cartilage contributed to regeneration. The results indicated that new hyaline cartilage could regenerate around the site of the injury. However, strange ectopic ossification could be found in the regeneration region (Liang et al., 2014; Yamagata et al., 2018). With a better understanding of the roles of MSCs in distraction histogenesis, accumulating evidences have shown that their beneficial role depends on their release of paracrine factors, especially through secreted exosomes (Borger et al., 2017; Liang et al., 2014; Shen et al., 2013). Toh and others (2016) demonstrated that these secreted exosomes could promote cartilage regeneration through intra-articular injection of ESC-exos into the rat model every week.

Another group combined hiPSC-MSC-exos with photoinduced imine crosslinking (PIC) hydrogels to form acellular tissue patches (EHGs) that could fix exosomes and exert a positive role in cellular regulation. They implanted EHGs into the cartilage-deficient site in a rabbit model and after 12 weeks found that the EHGs could accurately permeate and repair the cartilage defects (Liu et al., 2017b). A recent study found that the chondrocyte regenerationrelated genes SOX9 and WNT7A were upregulated by BM- 
MSC-exo transplantation. In addition, after coculture with chondrocytes, BM-MSC-exos significantly downregulated the expression of genes involved in hypertrophic chondrocyte differentiation, such as RUNX2, COL10A1, and ALP (Vonk et al., 2018). Therefore, MSC-exos could promote cartilage regeneration in $\mathrm{OA}$.

\section{MSC-exos modulate synovial inflammation in $O A$}

The destruction of the cartilage matrix is one of the paramount hallmarks of OA, while effects on the synovium are considered an important sign during the pathological process. A large amount of data has revealed that synovial inflammation appears not only in the late phase but also in the early phase of OA (van Lent et al., 2012). Inflammation is closely related to the joint destruction, pain, and suffering. The synovium consists of macrophages, fibroblast-like synoviocytes, and endothelial cells. These cells could actively participate in inflammatory reactions in an OA model (de Munter et al., 2016).

Synovial membranes that produce lubricin and $\mathrm{HC}$ can secrete synovial fluid, which contributes to the typical properties of articular cells and regulates chondrocyte activity. Once the synovial membrane undergoes pathological changes such as inflammation and hyperplasia, the volume and concentration of synovial fluid will be altered (Scanzello and Goldring, 2012). Synovial membranes also secrete pro-inflammation cytokines and catabolic agents, including IL- $1 \mathrm{~s}$, MMPs, and aggrecanases. IL- $1 \beta$ is the most significant catabolic cytokine. Several studies demonstrated that OA chondrocytes and synoviocytes expressed MMP-13 at a high level in response to IL- $1 \beta$ (Genemaras et al., 2016; Yamasaki et al., 2009).

When cartilage degraded, cartilage fragments dispersed over the articular cavity. Synovial cells could contact cartilage fragments and produce inflammatory mediators. These mediators not only activate metalloproteinase synthesis but also induce synovial angiogenesis and promote the expression of inflammatory cytokines (Berenbaum, 2012).

MSCs have tremendous potential for antifibrotic therapy, damage repair, and antiapoptotic therapy. Recently, many scientists have focused on the anti-inflammatory functions of MSCs. MSCs can obtain immunosuppressive properties and then activate the adaptive and innate immune systems when exposed to an inflammatory microenvironment. In addition, MSCs can promote the transformation of classically activated macrophages into alternately activated macrophages, inhibiting the activation of natural killer cells (Abumaree et al., 2013). In a model of early-phase OA, intra-articular injection of ADSCs could inhibit synovial thickening and protect cartilage destruction through the release of inflammatory factors from the synovium (ter Huurne et al., 2012).

Recently, it was found that intra-articular injection of MSCs could reduce the sensitivity to pain and enhance the release of anti-inflammatory factors (Ichiseki et al., 2018). Exosomes, as carriers from their parental cells, can communicate with other cells through ligands or other molecular signals. MSCs are able to suppress the local immune system and apoptosis in OA joints through paracrine activities (Caplan and Dennis, 2006). MSC-exos have been shown to exert immunosuppressive effects in many diseases. However, there are few reports on the anti-inflammatory effects of MSCs in OA models. One study showed that mature DCexos could accelerate endothelial inflammation through membrane TNF- $\alpha$ triggered by the NF- $\kappa \beta$ pathway (Gao et al., 2016).

In addition, MSC-exos can inhibit the pro-inflammatory cytokines IL- $1 \beta$, IL- 6 , and TNF- $\alpha$. Furthermore, exosomes might induce the transformation of Th1 cells into Th2 cells and suppress their ability to differentiate into Th17 cells (Chen et al., 2016b). In general, previous reports have indicated that the immunosuppressive properties of MSCs may be partly mediated by their release of exosomes that contain immune regulatory factors. However, the underlying mechanism needs further investigation.

\section{MSC-exos regulate bone metabolism in $O A$}

In addition to the degeneration of cartilage, the synovium, and the cartilage ECM, effects on the subchondral bone also exert a vital role in OA progression (Li and Aspden, 1997). Subchondral bones play a crucial role in the joint under the articular cartilage, including the cortical endplate and the trabecular bone. The main function of the subchondral bone is to absorb stress, supply nutrition to cartilage, and maintain the joint shape.

Magnetic resonance imaging has proven some alterations of subchondral bone, including calcified cartilage thickening, subchondral bone sclerosis, tidemark duplication, and appearance of osteophytes during OA (Henrotin et al., 2012). These changes have become a major medical problem due not only to their high incidence rate but also to their high associated medical expenses (Lee et al., 2018). The way to solve the above problems is to balance osteoclastmediated bone resorption and osteoblast-mediated bone formation (Rho et al., 2004). However, bone remodeling is a complicated process involving many signaling pathways and cytokines, such as TGF- $\beta$ signaling and IL-6 and calcitonin pathways (Karsdal et al., 2008).

The variations in these pathways begin at the subchondral surface, where pro-inflammatory or excessive mechanical stress initiates osteoclast-mediated bone resorption. Bone resorption is regulated by three key mediators, including OPG, RANK, and RANKL (Charles and Aliprantis, 2014). Inside the human airtight joint cavity, pro-inflammatory factors and biomechanical factors can affect the metabolism of subchondral bone. IL- $1 \beta$ and $\mathrm{TNF}-\alpha$ are considered to upregulate osteoclast-mediated bone resorption but downregulate osteoblast-mediated bone formation and differentiation (Lencel and Magne, 2011). One group found that the increase in osteoclasts in early OA was closely related to the appearance and persistence of sensory nerves in the subchondral bone, demonstrating the role of osteoclast-derived Netrin-1 in mediating OA pain (Zhu et al., 2019).

Abnormal biomechanical factors, including obesity, trauma, and abnormal anatomy, may induce OA (Guilak, 2011). However, it is still unclear whether the variations in subchondral bone in OA are pathological changes or normal physiological processes.

A large number of clinical and nonclinical studies have investigated the connections between subchondral bone and cartilage degradation. Some studies have demonstrated that 
calcitonin, strontium ranelate, and bisphosphonates can attenuate bone remodeling. A phase 3 clinical trial evaluated the structural changes through determination of cartilage volume loss and body mass index changes. Treatment with SrRan 2 g/day was found to have beneficial effects on such parameters in the knee OA patients (Pelletier et al., 2015). Adults aged $50-80$ years $(n=59)$ with clinical knee OA and knee bone marrow lesions (BMLs) were randomized to receive either zoledronic acid $(\mathrm{ZA})(5 \mathrm{mg} / 100 \mathrm{~mL})$ or placebo. The results indicated that ZA reduced knee pain and areal
BML size and increased the recovery proportion over 6 months (Laslett et al., 2012).

However, most clinical trials have acquired appealing outcomes, regardless of unideal experimental designs and unpredictable side effects. A novel therapeutic approach, rather than pharmacological treatments, could amend the joint structural alterations. Many groups had confirmed that MSC can alleviate OA. Recent reports demonstrated that MSC-based OA therapy could amend subchondral bone progression (Pers et al., 2015).

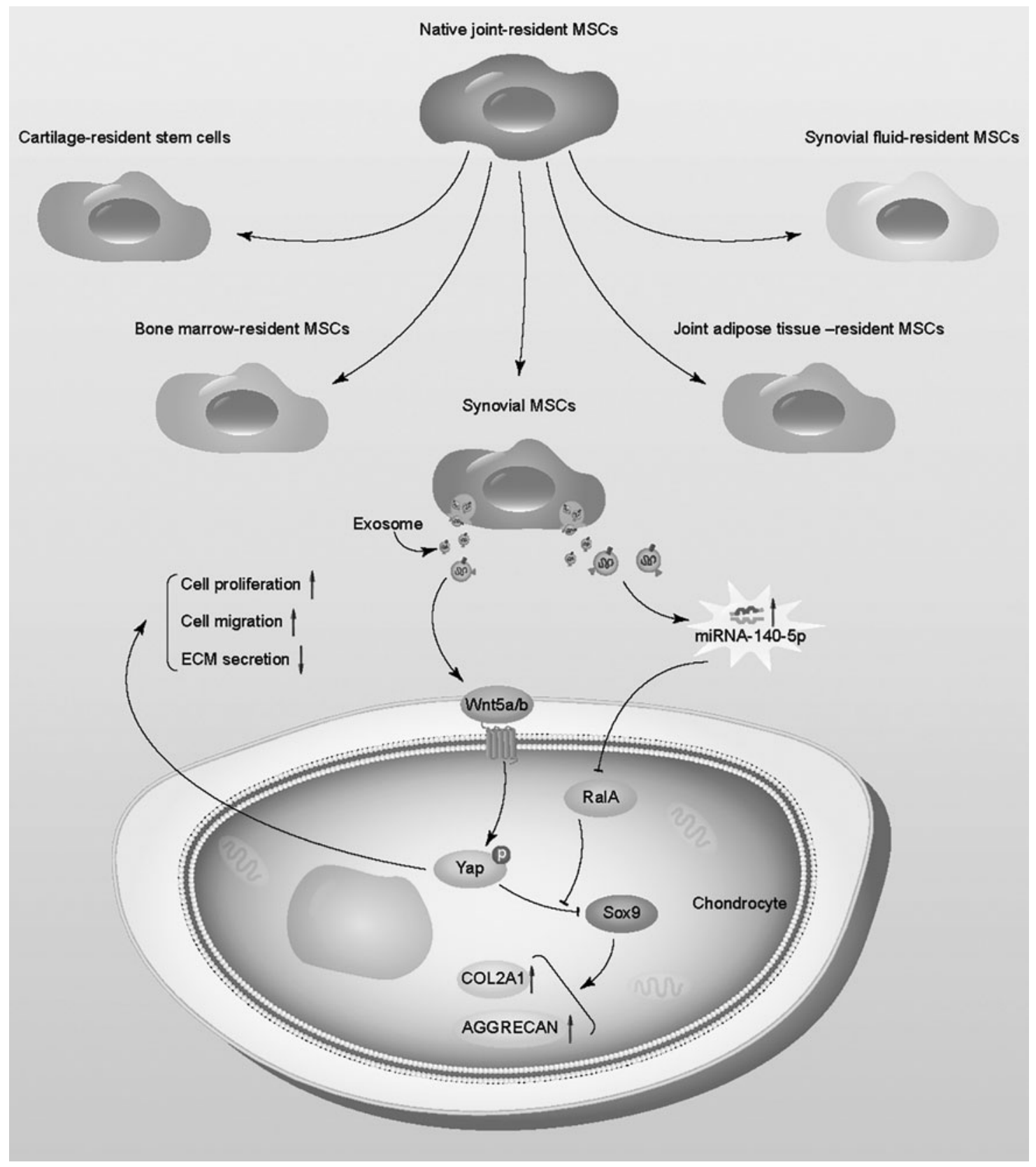

FIG. 2. Multiple native joint-resident stem cells in the joint. Native stem cells are abundant among the joint cavity, including cartilage, bone marrow, synovium, joint adipose, and synovial fluid. Such stem cells may act a protective strategy in osteoarthritic chondrocytes. Synovial MSCs exhibit superior chondrogenic ability compared with other native jointresident stem cells. Exosomes derived from miR-140-5p overexpressing synovial stem cells could enhance the proliferation and migration of articular chondrocytes through Wnt signaling pathways and block the side effect through RalA signaling pathways. MSC, mesenchymal stem cell. 
Many scientists have attributed the therapeutic effect to mediators such as exosomes. Exosomes can transmit their contents to cells, resulting in many cellular activities, such as cell proliferation, differentiation, and even cell death. One study suggested that extracellular vesicles from ADSCs, which downregulated inflammation and oxidative stress, may lead to antisenescence effects in OA osteoblasts (Tofino-Vian et al., 2017). It has been demonstrated that BM-MSC-derived exosomes could accelerate osteoclastogenesis in alveolar bone deterioration. Vascular endothelial cell-derived exosomes can attenuate bone resorption in vitro and in vivo through miRNA-155 upregulation (Song et al., 2019).

However, there are few reports on the relationship between exosomes derived from MSCs and bone metabolism in OA. Recent reports revealed that the information exchange between the native joint-resident stem cells involved in this coordinated process played a pivotal role in maintaining bone homeostasis and self-repair (McGonagle et al., 2017; Nakahama, 2010) (Fig. 2). Clinical studies have reported that the accumulation of mesenchymal progenitor cells in joint tissues and synovial fluids is accompanied by the progression of OA (Koyama et al., 2011; Sekiya et al., 2012). In the anterior cruciate ligament transection (ACLT) model, knockout of Tgfbr2 in nestin-positive MSCs attenuated the progression of OA (Zhen et al., 2013). Taken together, these results highlight that native joint-resident stem cells can prevent the development of arthritis.

There are perforations and blood vessels between the subchondral bone and cartilage. Through these channels, biomolecules and hormones can be exchanged (Walsh et al., 2010). Perhaps exosomes can affect the development of OA by acting on native joint-resident stem cells through such channels, but further evidence is needed urgently.

\section{Exosomes' miRNA in the OA}

Many studies have reported the miRNA profile of MSCs by comparing physiological and pathological states (Lakshmipathy and Hart, 2008; Vicente et al., 2016). They attempted to determine the relationship between miRNAs and the regulatory pathways of OA. microRNAs, a kind of noncoding RNA, can negatively regulate the expression of many target genes by promoting mRNA degradation and repressing translation. These single-stranded RNAs participate in chondrocyte differentiation and regulate ECM remodeling. miRNA-181b exerts a negative regulatory effect on chondrocyte in OA (Song et al., 2013).

miRNA-320 could regulate cartilage development and chondrogenesis by directly targeting MMP-13 (Meng et al., 2016). Meanwhile, a group suggested that miRNA-145 could regulate human chondrocyte function by directly inhibiting SOX9 (Martinez-Sanchez et al., 2012). EVmiRNA, a database of miRNA profiles in extracellular vesicles, gathered 462 miRNA expression profiles from extracellular vesicles (EVs) in 17 tissues/diseases. This database could help us to better understand the role of EVmiRNA in the treatment and diagnosis of diseases (Liu et al., 2019). miRNAs existing in exosomes also have the capacity to influence the surrounding cells through intercellular communication.

The exosome membrane maintains the classic topology of the plasma membrane and envelopes specific cytoplasmic cargo such as miRNAs, IncRNAs, mRNAs, and proteins (Valadi et al., 2007). Therefore, MSC-exos may affect OA progression through miRNA regulation. Numerous studies have reported that MSC-exos exert beneficial effects through specific miRNAs. One study suggests that MSCexos may reverse the pathological inflammatory condition without causing further significant toxicity through three specific miRNAs: miRNA21, miRNA-146a, and miRNA181c (Ti et al., 2016). Exosomal miRNA-125a could regulate endothelial tip cells by targeting DLL4 to promote angiogenesis. This finding provides a novel perspective on the relationship between MSCs and endothelial cells in physiological and pathological processes (Liang et al., 2016).

miRNA-100-5p inhibited mTOR-autophagy pathway and suppressed chondrocyte apoptosis by balancing anabolic and catabolic processes (Wu et al., 2019). In a rat model, MSCexo-derived miRNA-135b can promote cartilage repair by
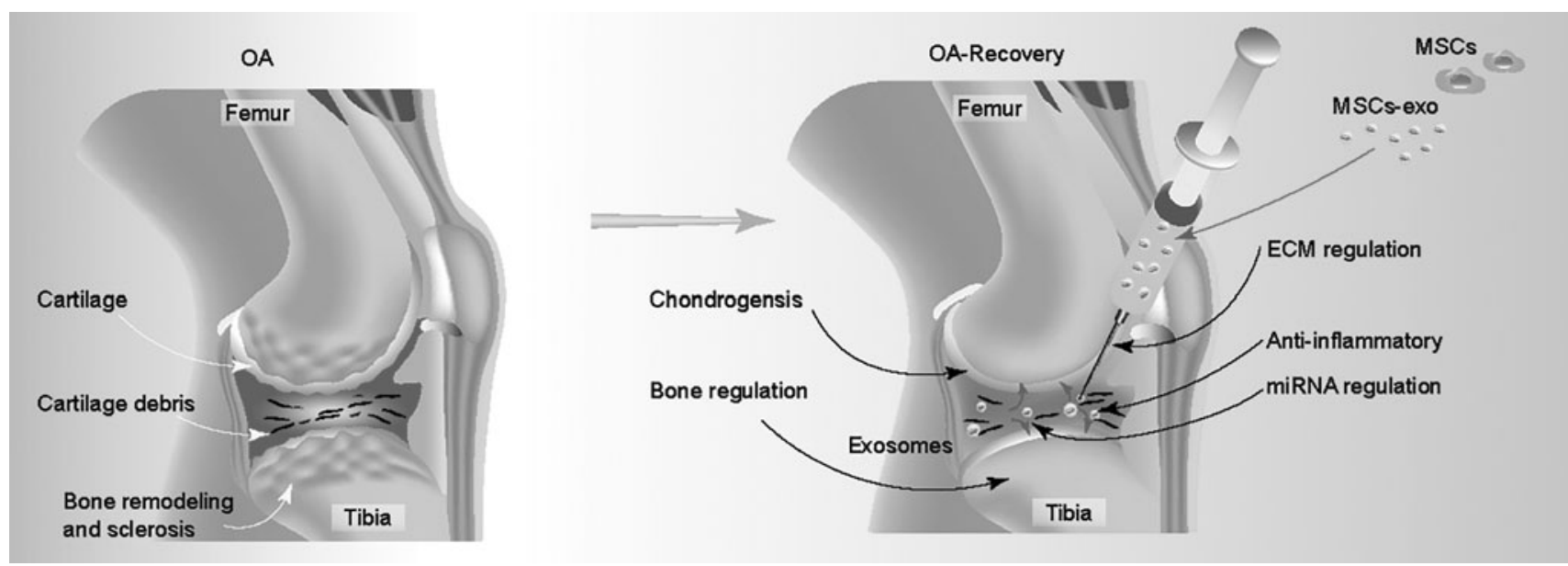

FIG. 3. Compare OA with OA recovery. OA is a progressive and heterogeneous disease, which contributes to articular cartilage degeneration, alterations in subchondral bone synovial hyperplasia (left); MSC-exo can regulate the chondrogenesis, bone remodeling, ECM, and miRNA and also anti-inflammatory effects (right). ECM, extracellular matrix; OA, Osteoarthritis. 
regulating TGF- $\beta$ (Wang et al., 2018). Exosomes derived from miRNA-140-5p-overexpressing human synovial MSCs (hSMSCs) could promote cartilage tissue regeneration and block the side effects of OA by regulating RalA (Fig. 2) (Tao et al., 2017). These miRNAs could specifically target immune-related genes involved in transcription, transactivation, and epigenetic modification (Ti et al., 2016).

IL-6 and TLRs, two critical factors, have already been reported to mediate MSC-exos with immune activation. Therefore, miRNAs derived from MSC-exos may regulate immune homeostasis through IL-6 and TLRs. However, the miRNA content in exosomes is relatively low, and miRNA quantity is influenced by cell type and cell viability and cell environment. One study showed that human embryonic stem cell exosomes were rich in pre-miRNAs.

In contrast, another group demonstrated that adult MSCs contained more mature miRNAs than immature MSCs. The maturation mechanism of miRNAs may vary with cell types and cell microenvironment (Baglio et al., 2015). Overall, MSC-exo-derived miRNA can promote the proliferation of chondrocyte, attenuate apoptosis, and regulate the immune response. Understanding the underlying mechanisms might provide some new directions for the treatment of OA.

\section{Conclusions and Perspectives}

In recent decades, it has been broadly reported that MSCexos could promote articular cartilage regeneration, inhibit inflammatory reactions, and regulate bone metabolism in OA. MSC-exos induce cartilage regeneration by promoting angiogenesis and inhibiting chondrocyte hypertrophy. The miRNAs and proteins in the exosomes play an important role. Therefore, exosome-based therapy has considerable potential as a stem cell therapy.

In this review, we concentrated on exosome functions in different aspects of articular OA (Fig. 3). Furthermore, our review also conveys novel insight into the role of exosome derived miRNAs in promoting cell proliferation and migration, as well as chondrogenesis and angiogenesis. However, the underlying mechanism needs further investigation before strategies can be applied to clinical therapy. Exosomes are widely used for disease prediction and screening. The lipid bilayer of the exosome membrane can protect its contents from enzyme digestion in different physical conditions, allowing exosome to communicate with essential signaling pathways. Making use of this communication overcomes the problems of tumorigenicity and cytotoxicity inherent with cell-based therapy. More importantly, exosomes can cross the blood-brain barrier and have low immunogenicity.

Thus, the feasibility of using exosomes for drug delivery offers great potential in the development of safe and effective therapies for degenerative disease. However, obtaining exosomes with higher purity and quality is limited by the current state of technology, stem cell type, and price. Cross talk between joint-resident stem cells and the transplanted stem cells is an important communication pathway. Exosomes surely play an indispensable role in this pathway. Current and future work requires the use of exosome-based OA therapies that have been proposed before clinical applications to solve various problems. In general, MSC-exo-based treatments may represent a new strategy to alleviate matrix degradation and anti-inflammatory functions in OA models.

\section{Author Disclosure Statement}

The authors declare they have no conflicting financial interests.

\section{Funding Information}

This study was supported by The National Major Scientific and Technological Special Project for "Significant New Drugs Development" (2018ZX09201002-005); Shanghai Science and Technology Commission International Cooperation Project on Science and Technology Innovation (18410760100); Key Science and Technology Innovation Projects of the Ministry of Science and Technology (2017YFE0121100); Xu Jun's expert work station (2017IC025); and the Fundamental Research Fund for the Central Universities (1500219107, J.X.).

\section{References}

Abumaree, M.H., Al Jumah, M.A., Kalionis, B., Jawdat, D., Al Khaldi, A., Abomaray, F.M., Fatani, A.S., Chamley, L.W., and Knawy, B.A. (2013). Human placental mesenchymal stem cells (pMSCs) play a role as immune suppressive cells by shifting macrophage differentiation from inflammatory M1 to anti-inflammatory M2 macrophages. Stem Cell Rev. 9, 620-641.

Ailawadi, S., Wang, X., Gu, H., and Fan, G.C. (2015). Pathologic function and therapeutic potential of exosomes in cardiovascular disease. Biochim. Biophys. Acta 1852, 1-11.

Allen, K. D., and Golightly, Y.M. (2015). State of the evidence. Curr. Opin. Rheumatol. 27, 276-283.

Baglio, S.R., Rooijers, K., Koppers-Lalic, D., Verweij, F.J., Perez Lanzon, M., Zini, N., Naaijkens, B., Perut, F., Niessen, H.W., Baldini, N., and Pegtel, D.M. (2015). Human bone marrow- and adipose-mesenchymal stem cells secrete exosomes enriched in distinctive miRNA and tRNA species. Stem Cell Res. Ther. 6, 127.

Berenbaum, F. (2012). Osteoarthritis as an inflammatory disease (osteoarthritis is not osteoarthrosis!). Osteoarthritis Cartilage 21, 16-21.

Blanchard, N., Lankar, D., Faure, F., Regnault, A., Dumont, C., Raposo, G., and C. Hivroz. (2002). TCR activation of human $\mathrm{T}$ cells induces the production of exosomes bearing the TCR/CD3/zeta complex. J. Immunol. 168, 3235-3241.

Böing, A.N., van der Pol, E., Grootemaat, A.E., Coumans, F.A.W., Sturk, A., and Nieuwland, R. (2014). Single-step isolation of extracellular vesicles by size-exclusion chromatography. J. Extracell. Vesicles 3, DOI: 10.3402/jev.v3.23430.

Borger, V., Bremer, M., Ferrer-Tur, R., Gockeln, L., Stambouli, O., Becic, A., and Giebel, B. (2017). Mesenchymal stem/ stromal cell-derived extracellular vesicles and their potential as novel immunomodulatory therapeutic agents. Int. J. Mol. Sci. 18, E1450.

Caplan, A. I., and Dennis, J.E. (2006). Mesenchymal stem cells as trophic mediators. J. Cell Biochem. 98, 1076-1084.

Charles, J. F., and Aliprantis, A.O. (2014). Osteoclasts: more than 'bone eaters'. Trends Mol. Med. 20, 449-459.

Chen, C., Zong, S., Wang, Z., Lu, J., Zhu, D., Zhang, Y., and Cui, Y. (2016a). Imaging and intracellular tracking of cancerderived exosomes using single-molecule localization-based super-resolution microscope. ACS Appl. Mater Interfaces 8, 25825-25833.

Chen, W., Huang, Y., Han, J., Yu, L., Li, Y., Lu, Z., Li, H., Liu, Z., Shi, C., Duan, F., and Xiao, Y. (2016b). Immunomodu- 
latory effects of mesenchymal stromal cells-derived exosome. Immunol. Res. 64, 831-840.

Chiang, E.R., Ma, H.L., Wang, J.P., Liu, C.L., Chen, T.H., and Hung, S.C. (2016). Allogeneic mesenchymal stem cells in combination with hyaluronic acid for the treatment of osteoarthritis in rabbits. PLoS One 11, e0149835.

de Munter, W., van den Bosch, M.H., Sloetjes, A.W., Croce, K.J., Vogl, T., Roth, J., Koenders, M.I., van de Loo, F.A., van den Berg, W.B., van der Kraan, P.M., and van Lent, P.L. (2016). High LDL levels lead to increased synovial inflammation and accelerated ectopic bone formation during experimental osteoarthritis. Osteoarthritis Cartilage 24, 844-855.

Ding, M., Wang, C., Lu, X., Zhang, C., Zhou, Z., Chen, X., Zhang, C.Y., Zen, K., and Zhang, C. (2018). Comparison of commercial exosome isolation kits for circulating exosomal microRNA profiling. Anal. Bioanal. Chem. 410, 3805-3814.

Gao, L., Wang, L., Dai, T., Jin, K., Zhang, Z., Wang, S., Xie, F., Fang, P., Yang, B., Huang, H., van Dam, H., Zhou, F., and Zhang, L. (2018). Tumor-derived exosomes antagonize innate antiviral immunity. Nat. Immunol. 19, 233-245.

Gao, W., Liu, H., Yuan, J., Wu, C., Huang, D., Ma, Y., Zhu, J., Ma, L., Guo, J., Shi, H., Zou, Y., and Ge, J. (2016). Exosomes derived from mature dendritic cells increase endothelial inflammation and atherosclerosis via membrane TNF-alpha mediated NF-kappaB pathway. J. Cell Mol. Med. 20, 2318 2327.

Genemaras, A.A., Ennis, H., Kaplan, L., and Huang, C.Y. (2016). Inflammatory cytokines induce specific time- and concentration-dependent MicroRNA release by chondrocytes, synoviocytes, and meniscus cells. J. Orthop. Res. 34, 779790.

Gnecchi, M., Danieli, P., Malpasso, G., and Ciuffreda, M.C. (2016). Paracrine mechanisms of mesenchymal stem cells in tissue repair. Methods Mol. Biol. 1416, 123-146.

Guilak, F. (2011). Biomechanical factors in osteoarthritis. Best Pract. Res. Clin. Rheumatol. 25, 815-823.

Henrotin, Y., Pesesse, L., and Sanchez, C. (2012). Subchondral bone and osteoarthritis: biological and cellular aspects. Osteoporos Int. 23(Suppl. 8), S847-851.

Iacopetta, B.J., Morgan, E.H., and Yeoh, G.C. (1983). Receptor-mediated endocytosis of transferrin by developing erythroid cells from the fetal rat liver. J. Histochem. Cytochem. 31, 336-344.

Ichiseki, T., Shimazaki, M., Ueda, Y., Ueda, S., Tsuchiya, M., Souma, D., Kaneuji, A., and Kawahara, N. (2018). Intraarticularly-injected mesenchymal stem cells stimulate anti-inflammatory molecules and inhibit pain related protein and chondrolytic enzymes in a monoiodoacetate-induced rat arthritis model. Int. J. Mol. Sci. 19, E203.

Iliopoulos, D., Malizos, K.N., Oikonomou, P., and Tsezou, A. (2008). Integrative microRNA and proteomic approaches identify novel osteoarthritis genes and their collaborative metabolic and inflammatory networks. PLoS One 3, e3740.

Jo, C.H., Lee, Y.G., Shin, W.H., Kim, H., Chai, J.W., Jeong, E.C., Kim, J.E., Shim, H., Shin, J.S., Shin, I.S., Ra, J.C., Oh, S., and Yoon, K.S. (2014). Intra-articular injection of mesenchymal stem cells for the treatment of osteoarthritis of the knee: a proof-of-concept clinical trial. Stem Cells 32, 1254-1266.

Johnstone, R.M., Adam, M., Hammond, J.R., Orr, L., and Turbide, C. (1987). Vesicle formation during reticulocyte maturation. Association of plasma membrane activities with released vesicles (exosomes). J. Biol. Chem. 262, 9412-9420.
Karsdal, M.A., Leeming, D.J., Dam, E.B., Henriksen, K., Alexandersen, P., Pastoureau, P., Altman, R.D., and Christiansen, C. (2008). Should subchondral bone turnover be targeted when treating osteoarthritis? Osteoarthritis Cartilage 16, 638-646.

Kordelas, L., Rebmann, V., Ludwig, A.K., Radtke, S., Ruesing, J., Doeppner, T.R., Epple, M., Horn, P.A., Beelen, D.W., and Giebel, B. (2014). MSC-derived exosomes: a novel tool to treat therapy-refractory graft-versus-host disease. Leukemia 28, 970-973.

Koyama, N., Okubo, Y., Nakao, K., Osawa, K., Fujimura, K., and Bessho, K. (2011). Pluripotency of mesenchymal cells derived from synovial fluid in patients with temporomandibular joint disorder. Life Sci. 89, 741-747.

Kuroda, K., Kabata, T., Hayashi, K., Maeda, T., Kajino, Y., Iwai, S., Fujita, K., Hasegawa, K., Inoue, D., Sugimoto, N., and Tsuchiya, H. (2015). The paracrine effect of adiposederived stem cells inhibits osteoarthritis progression. BMC Musculoskelet. Disord. 16, 236.

Lakshmipathy, U., and Hart, R.P. (2008). Concise review: microRNA expression in multipotent mesenchymal stromal cells. Stem Cells 26, 356-363.

Laslett, L.L., Dore, D.A., Quinn, S.J., Boon, P., Ryan, E., Winzenberg, T.M., and Jones, G. (2012). Zoledronic acid reduces knee pain and bone marrow lesions over 1 year: a randomised controlled trial. Ann. Rheum. Dis. 71, 13221328.

Lee, S.Y., Kim, G.T., Yun, H.M., Kim, Y.C., Kwon, I.K., and Kim, E.C. (2018). Tectorigenin promotes osteoblast differentiation and in vivo bone healing, but suppresses osteoclast differentiation and in vivo bone resorption. Mol. Cells 41, 476-485.

Leibacher, J., and Henschler, R. (2016). Biodistribution, migration and homing of systemically applied mesenchymal stem/stromal cells. Stem Cell Res. Ther. 7, 7.

Lencel, P., and Magne, D. (2011). Inflammaging: the driving force in osteoporosis? Med. Hypotheses 76, 317-321.

Li, B., and Aspden, R.M. (1997). Mechanical and material properties of the subchondral bone plate from the femoral head of patients with osteoarthritis or osteoporosis. Ann. Rheum. Dis. 56, 247-254.

Liang, L.G., Sheng, Y.F., Zhou, S., Inci, F., Li, L., Demirci, U., and Wang, S. (2017). An integrated double-filtration microfluidic device for detection of extracellular vesicles from urine for bladder cancer diagnosis. Methods Mol. Biol. 1660, 355-364.

Liang, X., Ding, Y., Zhang, Y., Tse, H.F., and Lian, Q. (2014). Paracrine mechanisms of mesenchymal stem cell-based therapy: current status and perspectives. Cell Transplant. 23, 1045-1059.

Liang, X.L., Zhang, L.N., Wang, S.H., Han, Q., and Zhao, R.C. (2016). Exosomes secreted by mesenchymal stem cells promote endothelial cell angiogenesis by transferring miR-125a. J. Cell Sci. 129, 2182-2189.

Liu, C., Guo, J., Tian, F., Yang, N., Yan, F., Ding, Y., Wei, J., $\mathrm{Hu}$, G., Nie, G., and Sun, J. (2017a). Field-free isolation of exosomes from extracellular vesicles by microfluidic viscoelastic flows. ACS Nano 11, 6968-6976.

Liu, T., Zhang, Q., Zhang, J., Li, C., Miao, Y.R., Lei, Q., Li, Q., and Guo, A.Y. (2019). EVmiRNA: a database of miRNA profiling in extracellular vesicles. Nucleic Acids Res. 47, D89-D93.

Liu, X., Yang, Y., Li, Y., Niu, X., Zhao, B., Wang, Y., Bao, C., Xie, Z., Lin, Q., and Zhu, L. (2017b). Integration of stem cell- 
derived exosomes with in situ hydrogel glue as a promising tissue patch for articular cartilage regeneration. Nanoscale 9, 4430-4438.

Lories, R.J. (2008). Joint homeostasis, restoration, and remodeling in osteoarthritis. Best Pract. Res. Clin. Rheumatol. 22, 209-220.

Lotvall, J., Hill, A.F., Hochberg, F., Buzas, E.I., Di Vizio, D., Gardiner, C., Gho, Y.S., Kurochkin, I.V., Mathivanan, S., Quesenberry, P., Sahoo, S., Tahara, H., Wauben, M.H., Witwer, K.W., and Thery, C. (2014). Minimal experimental requirements for definition of extracellular vesicles and their functions: a position statement from the International Society for Extracellular Vesicles. J. Extracell. Vesicles 3, 26913.

Ludwig, A.K., and Giebel, B. (2012). Exosomes: small vesicles participating in intercellular communication. Int. J. Biochem. Cell Biol. 44, 11-15.

Makris, E.A., Gomoll, A.H., Malizos, K.N., Hu, J.C., and Athanasiou, K.A. (2015). Repair and tissue engineering techniques for articular cartilage. Nat. Rev. Rheumatol. 11, 21-34.

Martel-Pelletier, J., Barr, A.J., Cicuttini, F.M., Conaghan, P.G., Cooper, C., Goldring, M.B., Goldring, S.R., Jones, G., Teichtahl, A.J., and Pelletier, J.-P. (2016). Osteoarthritis. Nat. Rev. Dis. Primers 2, 16072.

Martinez-Sanchez, A., Dudek, K.A., and Murphy, C.L. (2012). Regulation of human chondrocyte function through direct inhibition of cartilage master regulator SOX9 by microRNA145 (miRNA-145). J. Biol. Chem. 287, 916-924.

McGonagle, D., Baboolal, T.G., and Jones, E. (2017). Native joint-resident mesenchymal stem cells for cartilage repair in osteoarthritis. Nat. Rev. Rheumatol. 13, 719-730.

Meng, F., Zhang, Z., Chen, W., Huang, G., He, A., Hou, C., Long, Y., Yang, Z., Zhang, Z., and Liao, W. (2016). MicroRNA-320 regulates matrix metalloproteinase-13 expression in chondrogenesis and interleukin-1beta-induced chondrocyte responses. Osteoarthritis Cartilage 24, 932-941.

Momen-Heravi, F., Balaj, L., Alian, S., Mantel, P.Y., Halleck, A.E., Trachtenberg, A.J., Soria, C.E., Oquin, S., Bonebreak, C.M., Saracoglu, E., Skog, J., and Kuo, W.P. (2013). Current methods for the isolation of extracellular vesicles. Biol. Chem. 394, 1253-1262.

Nakahama, K. (2010). Cellular communications in bone homeostasis and repair. Cell. Mol. Life Sci. 67, 4001-4009.

Nakai, W., Yoshida, T., Diez, D., Miyatake, Y., Nishibu, T., Imawaka, N., Naruse, K., Sadamura, Y., and Hanayama, R. (2016). A novel affinity-based method for the isolation of highly purified extracellular vesicles. Sci. Rep. 6, 33935.

Pak, J., Lee, J.H., Kartolo, W.A., and Lee, S.H. (2016). Cartilage regeneration in human with adipose tissue-derived stem cells: current status in clinical implications. Biomed. Res. Int. 2016, 4702674

Pelletier, J.P., Roubille, C., Raynauld, J.P., Abram, F., Dorais, M., Delorme, P., and Martel-Pelletier, J. (2015). Diseasemodifying effect of strontium ranelate in a subset of patients from the Phase III knee osteoarthritis study SEKOIA using quantitative MRI: reduction in bone marrow lesions protects against cartilage loss. Ann. Rheum. Dis. 74, 422-429.

Pers, Y.M., Ruiz, M., Noel, D., and Jorgensen, C. (2015). Mesenchymal stem cells for the management of inflammation in osteoarthritis: state of the art and perspectives. Osteoarthritis Cartilage 23, 2027-2035.

Pittenger, M.F., Mackay, A.M., Beck, S.C., Jaiswal, R.K., Douglas, R., Mosca, J.D., Moorman, M.A., Simonetti, D.W., Craig, S., and Marshak, D.R. (1999). Multilineage potential of adult human mesenchymal stem cells. Science 284, 143-147.
Qin, Y., Sun, R., Wu, C., Wang, L., and Zhang, C. (2016). Exosome: a novel approach to stimulate bone regeneration through regulation of osteogenesis and angiogenesis. Int. J. Mol. Sci. 17, E712.

Rho, J., Takami, M., and Choi, Y. (2004). Osteoimmunology: interactions of the immune and skeletal systems. Mol. Cells 17, 1-9.

Roelofs, A.J., Rocke, J.P., and De Bari, C. (2013). Cell-based approaches to joint surface repair: a research perspective. Osteoarthritis Cartilage 21, 892-900.

Saw, K.Y., Anz, A., Siew-Yoke Jee, C., Merican, S., ChingSoong Ng, R., Roohi, S.A., and Ragavanaidu, K. (2013). Articular cartilage regeneration with autologous peripheral blood stem cells versus hyaluronic acid: a randomized controlled trial. Arthroscopy 29, 684-694.

Scanzello, C.R., and Goldring, S.R. (2012). The role of synovitis in osteoarthritis pathogenesis. Bone 51, 249-257.

Sekiya, I., Ojima, M., Suzuki, S., Yamaga, M., Horie, M., Koga, H., Tsuji, K., Miyaguchi, K., Ogishima, S., Tanaka, H., and Muneta. T. (2012). Human mesenchymal stem cells in synovial fluid increase in the knee with degenerated cartilage and osteoarthritis. J. Orthop. Res. 30, 943-949.

Sharma, A. (2018). Role of stem cell derived exosomes in tumor biology. Int. J. Cancer 142, 1086-1092.

Shen, L., Zeng, W., Wu, Y.X., Hou, C.L., Chen, W., Yang, M.C., Li, L., Zhang, Y.F., and Zhu, C.H. (2013). Neurotrophin-3 accelerates wound healing in diabetic mice by promoting a paracrine response in mesenchymal stem cells. Cell Transplant. 22, 1011-1021.

Song, H., Li, X., Zhao, Z., Qian, J., Wang, Y., Cui, J., Weng, W., Cao, L., Chen, X., Hu, Y., and Su, J. (2019). Reversal of osteoporotic activity by endothelial cell-secreted bone targeting and biocompatible exosomes. Nano Lett. 19, 30403048 .

Song, J., Lee, M., Kim, D., Han, J., Chun, C.H., and Jin, E.J. (2013). MicroRNA-181b regulates articular chondrocytes differentiation and cartilage integrity. Biochem Biophys. Res. Commun. 431, 210-214.

Tao, S.C., Yuan, T., Zhang, Y.L., Yin, W.J., Guo, S.C., and Zhang, C.Q. (2017). Exosomes derived from miR-140-5poverexpressing human synovial mesenchymal stem cells enhance cartilage tissue regeneration and prevent osteoarthritis of the knee in a rat model. Theranostics 7, 180-195.

ter Huurne, M., Schelbergen, R., Blattes, R., Blom, A., de Munter, W., Grevers, L.C., Jeanson, J., Noel, D., Casteilla, L., Jorgensen, C., van den Berg, W., and van Lent, P.L. (2012). Antiinflammatory and chondroprotective effects of intraarticular injection of adipose-derived stem cells in experimental osteoarthritis. Arthritis Rheum. 64, 3604-3613.

Thery, C., Witwer, K.W., Aikawa, E., Alcaraz, M.J., Anderson, J.D., Andriantsitohaina, R., Antoniou, A., Arab, T., Archer, F., Atkin-Smith, G.K., Ayre, D.C., Bach, J.M., Bachurski, D., Baharvand, H., Balaj, L., Baldacchino, S., Bauer, N.N., Baxter, A.A., Bebawy, M., Beckham, C., Bedina Zavec, A., Benmoussa, A., Berardi, A.C., Bergese, P., Bielska, E., Blenkiron, C., Bobis-Wozowicz, S., Boilard, E., Boireau, W., Bongiovanni, A., Borras, F.E., Bosch, S., Boulanger, C.M., Breakefield, X., Breglio, A.M., Brennan, M.A., Brigstock, D.R., Brisson, A., Broekman, M.L., Bromberg, J.F., BrylGorecka, P., Buch, S., Buck, A.H., Burger, D., Busatto, S., Buschmann, D., Bussolati, B., Buzas, E.I., Byrd, J.B., Camussi, G., Carter, D.R., Caruso, S., Chamley, L.W., Chang, Y.T., Chen, C., Chen, S., Cheng, L., Chin, A.R., Clayton, A., Clerici, S.P., Cocks, A., Cocucci, E., Coffey, R.J., Cordeiro- 
da-Silva, A., Couch, Y., Coumans, F.A., Coyle, B., Crescitelli, R., Criado, M.F., D’Souza-Schorey, C., Das, S., Datta Chaudhuri, A., de Candia, P., De Santana, E.F., De Wever, O., Del Portillo, H.A., Demaret, T., Deville, S., Devitt, A., Dhondt, B., Di Vizio, D., Dieterich, L.C., Dolo, V., Dominguez Rubio, A.P., Dominici, M., Dourado, M.R., Driedonks, T.A., Duarte, F.V., Duncan, H.M., Eichenberger, R.M., Ekstrom, K., El Andaloussi, S., Elie-Caille, C., Erdbrugger, U., Falcon-Perez, J.M., Fatima, F., Fish, J.E., FloresBellver, M., Forsonits, A., Frelet-Barrand, A., Fricke, F., Fuhrmann, G., Gabrielsson, S., Gamez-Valero, A., Gardiner, C., Gartner, K., Gaudin, R., Gho, Y.S., Giebel, B., Gilbert, C., Gimona, M., Giusti, I., Goberdhan, D.C., Gorgens, A., Gorski, S.M., Greening, D.W., Gross, J.C., Gualerzi, A., Gupta, G.N., Gustafson, D., Handberg, A., Haraszti, R.A., Harrison, P., Hegyesi, H., Hendrix, A., Hill, A.F., Hochberg, F.H., Hoffmann, K.F., Holder, B., Holthofer, H., Hosseinkhani, B., Hu, G., Huang, Y., Huber, V., Hunt, S., Ibrahim, A.G., Ikezu, T., Inal, J.M., Isin, M., Ivanova, A., Jackson, H.K., Jacobsen, S., Jay, S.M., Jayachandran, M., Jenster, G., Jiang, L., Johnson, S.M., Jones, J.C., Jong, A., JovanovicTalisman, T., Jung, S., Kalluri, R., Kano, S.I., Kaur, S., Kawamura, Y., Keller, E.T., Khamari, D., Khomyakova, E., Khvorova, A., Kierulf, P., Kim, K.P., Kislinger, T., Klingeborn, M., Klinke, 2nd, D.J., Kornek, M., Kosanovic, M.M., Kovacs, A.F., Kramer-Albers, E.M., Krasemann, S., Krause, M., Kurochkin, I.V., Kusuma, G.D., Kuypers, S., Laitinen, S., Langevin, S.M., Languino, L.R., Lannigan, J., Lasser, C., Laurent, L.C., Lavieu, G., Lazaro-Ibanez, E., Le Lay, S., Lee, M.S., Lee, Y.X.F., Lemos, D.S., Lenassi, M., Leszczynska, A., Li, I.T., Liao, K., Libregts, S.F., Ligeti, E., Lim, R., Lim, S.K., Line, A., Linnemannstons, K., Llorente, A., Lombard, C.A., Lorenowicz, M.J., Lorincz, A.M., Lotvall, J., Lovett, J., Lowry, M.C., Loyer, X., Lu, Q., Lukomska, B., Lunavat, T.R., Maas, S.L., Malhi, H., Marcilla, A., Mariani, J., Mariscal, J., Martens-Uzunova, E.S., Martin-Jaular, L., Martinez, M.C., Martins, V.R., Mathieu, M., Mathivanan, S., Maugeri, M., McGinnis, L.K., McVey, M.J., Meckes, D.G., Jr., Meehan, K.L., Mertens, I., Minciacchi, V.R., Moller, A., Moller Jorgensen, M., Morales-Kastresana, A., Morhayim, J., Mullier, F., Muraca, M., Musante, L., Mussack, V., Muth, D.C., Myburgh, K.H., Najrana, T., Nawaz, M., Nazarenko, I., Nejsum, P., Neri, C., Neri, T., Nieuwland, R., Nimrichter, L., Nolan, J.P., Nolte-'t Hoen, E.N., Noren Hooten, N., O'Driscoll, L., O'Grady, T., O’Loghlen, A., Ochiya, T., Olivier, M., Ortiz, A., Ortiz, L.A., Osteikoetxea, X., Ostergaard, O., Ostrowski, M., Park, J., Pegtel, D.M., Peinado, H., Perut, F., Pfaffl, M.W., Phinney, D.G., Pieters, B.C., Pink, R.C., Pisetsky, D.S., Pogge von Strandmann, E., Polakovicova, I., Poon, I.K., Powell, B.H., Prada, I., Pulliam, L., Quesenberry, P., Radeghieri, A., Raffai, R.L., Raimondo, S., Rak, J., Ramirez, M.I., Raposo, G., Rayyan, M.S., Regev-Rudzki, N., Ricklefs, F.L., Robbins, P.D., Roberts, D.D., Rodrigues, S.C., Rohde, E., Rome, S., Rouschop, K.M., Rughetti, A., Russell, A.E., Saa, P., Sahoo, S., Salas-Huenuleo, E., Sanchez, C., Saugstad, J.A., Saul, M.J., Schiffelers, R.M., Schneider, R., Schoyen, T.H., Scott, A., Shahaj, E., Sharma, S., Shatnyeva, O., Shekari, F., Shelke, G.V., Shetty, A.K., Shiba, K., Siljander, P.R., Silva, A.M., Skowronek, A., Snyder, 2nd, O.L., Soares, R.P., Sodar, B.W., Soekmadji, C., Sotillo, J., Stahl, P.D., Stoorvogel, W., Stott, S.L., Strasser, E.F., Swift, S., Tahara, H., Tewari, M., Timms, K., Tiwari, S., Tixeira, R., Tkach, M., Toh, W.S., Tomasini, R., Torrecilhas, A.C., Tosar, J.P., Toxavidis, V., Urbanelli, L., Vader, P., van Balkom,
B.W., van der Grein, S.G., Van Deun, J., van Herwijnen, M.J., Van Keuren-Jensen, K., van Niel, G., van Royen, M.E., van Wijnen, A.J., Vasconcelos, M.H., Vechetti, I.J., Jr., Veit, T.D., Vella, L.J., Velot, E., Verweij, F.J., Vestad, B., Vinas, J.L., Visnovitz, T., Vukman, K.V., Wahlgren, J., Watson, D.C., Wauben, M.H., Weaver, A., Webber, J.P., Weber, V., Wehman, A.M., Weiss, D.J., Welsh, J.A., Wendt, S., Wheelock, A.M., Wiener, Z., Witte, L., Wolfram, J., Xagorari, A., Xander, P., Xu, J., Yan, X., Yanez-Mo, M., Yin, H., Yuana, Y., Zappulli, V., Zarubova, J., Zekas, V., Zhang, J.Y., Zhao, Z., Zheng, L., Zheutlin, A.R., Zickler, A.M., Zimmermann, P., Zivkovic, A.M., Zocco, D., and Zuba-Surma, E.K. (2018). Minimal information for studies of extracellular vesicles 2018 (MISEV2018): a position statement of the International Society for Extracellular Vesicles and update of the MISEV2014 guidelines. J. Extracell. Vesicles 7, 1535750.

Ti, D., Hao, H., Fu, X., and Han, W. (2016). Mesenchymal stem cells-derived exosomal microRNAs contribute to wound inflammation. Sci. China Life Sci. 59, 1305-1312.

Tofino-Vian, M., Guillen, M.I., Perez Del Caz, M.D., Castejon, M.A., and Alcaraz, M.J. (2017). Extracellular vesicles from adipose-derived mesenchymal stem cells downregulate senescence features in osteoarthritic osteoblasts. Oxid. Med. Cell. Longev. 2017, 7197598.

Valadi, H., Ekström, K., Bossios, A., Sjöstrand, M., Lee, J.J., and Lötvall, J.O. (2007). Exosome-mediated transfer of mRNAs and microRNAs is a novel mechanism of genetic exchange between cells. Nat. Cell Biol. 9, 654.

van Lent, P.L., Blom, A.B., Schelbergen, R.F., Sloetjes, A., Lafeber, F.P., Lems, W.F., Cats, H., Vogl, T., Roth, J., and van den Berg, W.B. (2012). Active involvement of alarmins S100A8 and S100A9 in the regulation of synovial activation and joint destruction during mouse and human osteoarthritis. Arthritis Rheum. 64, 1466-1476.

Vicente, R., Noel, D., Pers, Y.M., Apparailly, F., and Jorgensen, C. (2016). Deregulation and therapeutic potential of microRNAs in arthritic diseases. Nat. Rev. Rheumatol. 12, 211-220.

Vonk, L.A., van Dooremalen, S.F.J., Liv, N., Klumperman, J., Coffer, P.J., Saris, D.B.F., and Lorenowicz, M.J. (2018). Mesenchymal stromal/stem cell-derived extracellular vesicles promote human cartilage regeneration in vitro. Theranostics 8, 906-920.

Walsh, D.A., McWilliams, D.F., Turley, M.J., Dixon, M.R., Franses, R.E., Mapp, P.I., and Wilson, D. (2010). Angiogenesis and nerve growth factor at the osteochondral junction in rheumatoid arthritis and osteoarthritis. Rheumatology (Oxford) 49, 1852-1861.

Wang, R., Xu, B., and Xu, H. (2018). TGF-beta1 promoted chondrocyte proliferation by regulating Sp1 through MSCexosomes derived miR-135b. Cell Cycle 17, DOI: 10.1080/ 15384101.2018.1556063.

Withrow, J., Murphy, C., Liu, Y., Hunter, M., Fulzele, S., and Hamrick, M.W. (2016). Extracellular vesicles in the pathogenesis of rheumatoid arthritis and osteoarthritis. Arthritis Res. Ther. 18, 286.

Witwer, K.W., Van Balkom, B.W.M., Bruno, S., Choo, A., Dominici, M., Gimona, M., Hill, A.F., De Kleijn, D., Koh, M., Lai, R.C., Mitsialis, S.A., Ortiz, L.A., Rohde, E., Asada, T., Toh, W.S., Weiss, D.J., Zheng, L., Giebel, B., and Lim, S.K. (2019). Defining mesenchymal stromal cell (MSC)derived small extracellular vesicles for therapeutic applications. J. Extracell. Vesicles 8, 1609206.

Wu, J., Kuang, L., Chen, C., Yang, J., Zeng, W.N., Li, T., Chen, H., Huang, S., Fu, Z., Li, J., Liu, R., Ni, Z., Chen, L., and 
Yang, L. (2019). miR-100-5p-abundant exosomes derived from infrapatellar fat pad MSCs protect articular cartilage and ameliorate gait abnormalities via inhibition of mTOR in osteoarthritis. Biomaterials 206, 87-100.

Yamagata, K., Nakayamada, S., and Tanaka, Y. (2018). Use of mesenchymal stem cells seeded on the scaffold in articular cartilage repair. Inflamm. Regen. 38, 4.

Yamasaki, K., Nakasa, T., Miyaki, S., Ishikawa, M., Deie, M., Adachi, N., Yasunaga, Y., Asahara, H., and Ochi, M. (2009). Expression of MicroRNA-146a in osteoarthritis cartilage. Arthritis Rheum. 60, 1035-1041.

Zhang, H., Freitas, D., Kim, H.S., Fabijanic, K., Li, Z., Chen, H., Mark, M.T., Molina, H., Martin, A.B., Bojmar, L., Fang, J., Rampersaud, S., Hoshino, A., Matei, I., Kenific, C.M., Nakajima, M., Mutvei, A.P., Sansone, P., Buehring, W., Wang, H., Jimenez, J.P., Cohen-Gould, L., Paknejad, N., Brendel, M., Manova-Todorova, K., Magalhaes, A., Ferreira, J.A., Osorio, H., Silva, A.M., Massey, A., Cubillos-Ruiz, J.R., Galletti, G., Giannakakou, P., Cuervo, A.M., Blenis, J., Schwartz, R., Brady, M.S., Peinado, H., Bromberg, J., Matsui, H., Reis, C.A., and Lyden, D. (2018). Identification of distinct nanoparticles and subsets of extracellular vesicles by asymmetric flow field-flow fractionation. Nat. Cell Biol. 20, 332343.

Zhang, S., Chu, W.C., Lai, R.C., Lim, S.K., Hui, J.H., and Toh, W.S. (2016). Exosomes derived from human embryonic mesenchymal stem cells promote osteochondral regeneration. Osteoarthritis Cartilage 24, 2135-2140.
Zhang, S., Teo, K.Y.W., Chuah, S.J., Lai, R.C., Lim, S.K., and Toh, W.S. (2019). MSC exosomes alleviate temporomandibular joint osteoarthritis by attenuating inflammation and restoring matrix homeostasis. Biomaterials 200, 35-47.

Zhen, G., Wen, C., Jia, X., Li, Y., Crane, J.L., Mears, S.C., Askin, F.B., Frassica, F.J., Chang, W., Yao, J., Carrino, J.A., Cosgarea, A., Artemov, D., Chen, Q., Zhao, Z., Zhou, X., Riley, L., Sponseller, P., Wan, M., Lu, W.W., and Cao, X. (2013). Inhibition of TGF-beta signaling in mesenchymal stem cells of subchondral bone attenuates osteoarthritis. Nat. Med. 19, 704-712.

Zhu, S., Zhu, J., Zhen, G., Hu, Y., An, S., Li, Y., Zheng, Q., Chen, Z., Yang, Y., Wan, M., Skolasky, R.L., Cao, Y., Wu, T., Gao, B., Yang, M., Gao, M., Kuliwaba, J., Ni, S., Wang, L., Wu, C., Findlay, D., Eltzschig, H.K., Ouyang, H.W., Crane, J., Zhou, F.Q., Guan, Y., Dong, X., and Cao, X. (2019). Subchondral bone osteoclasts induce sensory innervation and osteoarthritis pain. J. Clin. Invest. 129, 1076-1093.

Address correspondence to:

Shane Gao

Tongji University School of Medicine

East Hospital

Shanghai 200120

China

E-mail: sammygao2014@163.com 